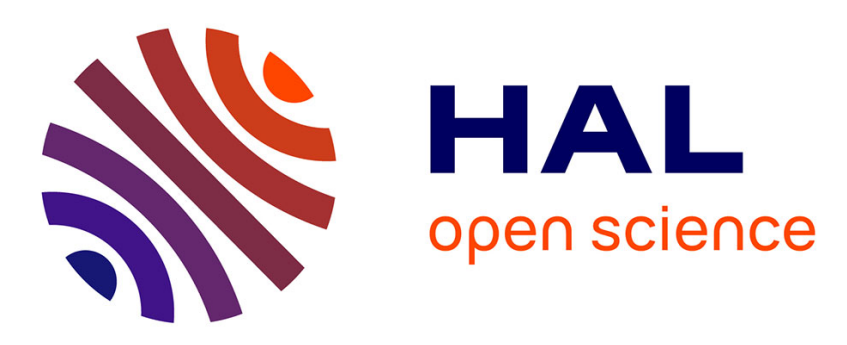

\title{
Photoacoustic sensor with radial and longitudinal acoustic resonators
}

Maxime Duquesnoy, Guillaume Aoust, Jean-Michel Melkonian, Raphaël Lévy, Myriam Raybaut, Antoine Godard

\section{- To cite this version:}

Maxime Duquesnoy, Guillaume Aoust, Jean-Michel Melkonian, Raphaël Lévy, Myriam Raybaut, et al.. Photoacoustic sensor with radial and longitudinal acoustic resonators. Laser Applications to Chemical, Security and Environmental Analysis 2020, Jun 2020, Washington, France. pp.LTh4F.5, 10.1364/LACSEA.2020.LTh4F.5 . hal-03152611

\section{HAL Id: hal-03152611 \\ https://hal.science/hal-03152611}

Submitted on 25 Feb 2021

HAL is a multi-disciplinary open access archive for the deposit and dissemination of scientific research documents, whether they are published or not. The documents may come from teaching and research institutions in France or abroad, or from public or private research centers.
L'archive ouverte pluridisciplinaire HAL, est destinée au dépôt et à la diffusion de documents scientifiques de niveau recherche, publiés ou non, émanant des établissements d'enseignement et de recherche français ou étrangers, des laboratoires publics ou privés. 


\title{
Photoacoustic sensor with radial and longitudinal acoustic resonators
}

\author{
Maxime Duquesnoy ${ }^{1,2}$, Guillaume Aoust ${ }^{1}$, Jean-Michel Melkonian ${ }^{2}$, \\ Raphaël Lévy $^{2}$, Myriam Raybaut ${ }^{2}$, Antoine Godard ${ }^{2}$ \\ ${ }^{1}$ Mirsense, Nanno-INNOV, building 863, 8 Avenue de la Vauve, 91120 Palaiseau, France \\ ${ }^{2}$ DPHY, ONERA, Université Paris Saclay, F-91123 Palaiseau, France \\ Author e-mail address: maxime.duquesnoy@mirsense.com
}

\begin{abstract}
We present a QEPAS sensor based on a custom quartz tuning fork associated with a radial acoustic resonator. We present a study through finite element method and demonstrate state of the art sensitivity. (C) 2020 The Authors
\end{abstract}

\section{Motivation}

Photoacoustic detectors have first been designed using microphones placed in acoustic resonators [1-3]. In 2002, a new technology emerged - called quartz enhanced photoacoustic spectroscopy (QEPAS) - using quartz tuning forks (QTF) instead of microphones for acoustic detection [4, 5].

In classical photoacoustic spectroscopy many works have already been carried out to determine the optimal geometry of the acoustic resonators to reach the best sensitivity [2, 3] (Figure 1a). In QEPAS, an acoustic resonator can be used to enhance the sensitivity, usually taking the form of tubes placed on each side of the QTF, inside which the laser passes. It is called on-beam configuration (Figure 1b). Despite the drawbacks of using these microresonators (high alignment sensitivity, diffraction of clipping of mid-infrared beams), there has been very little deviation from the longitudinal resonator geometry for QEPAS, an example of which is the off-beam configuration [6]. Besides, from the beginning of QEPAS, tuning forks for the clock industry have almost exclusively been used. A breakthrough has been made by Aoust \& al [7] and Spagnolo \& al [5], leading in both cases to QTFs with large prong spacing.

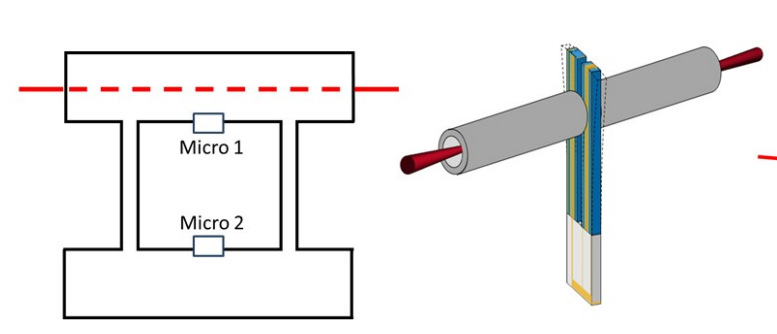

a) b)

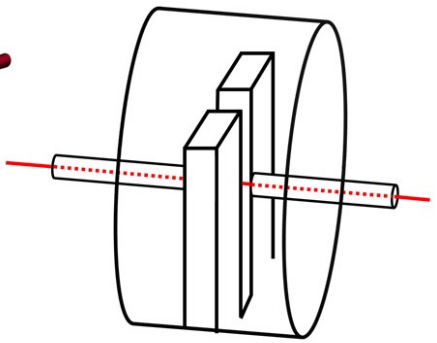

c)

Fig. 1. (a) Schematic of a differential Helmholtz resonator with microphones [8]. (b) Schematic of a QEPAS sensor in on-beam configuration, the tuning fork is surrounded by two longitudinal acoustic resonators in which the laser passes. (c) Schematic of our custom tuning fork with adapted longitudinal resonators as well as a cylindrical cavity for acoustic recovery.

\section{Our work}

We recently presented a custom QTF with adapted acoustic resonators [8, 10] (Figure 1c). This QTF, optimized to present the best quality factor in air at atmospheric standard conditions, is associated with an acoustic recovery cavity in order to avoid the important acoustic losses that would occur due to the emission of acoustic waves by the QTF's prongs. In order to enhance its sensitivity in a QEPAS experiment, the recovery cavity is coupled with adapted on-beam longitudinal resonators, enhancing the quality factor from 8000 to 75000 .

We now propose a new configuration for our custom QTF (similar to Figure 1c), where the acoustic cavity is made resonant through adaptation of its design. This resonator was modelled through finite element simulations (FEM) to study the influence of its geometry on the sensor's response (Figure 2). An optimal coupling with the QTF is obtained by modifying the resonator's radius. The quality factor is reduced from 94000 in our previous 
configuration to 600 in our new configuration when the maximum coupling is reached, and the signal-to-noise ratio is increased by a factor ten compared to our previous non-resonant cavity.
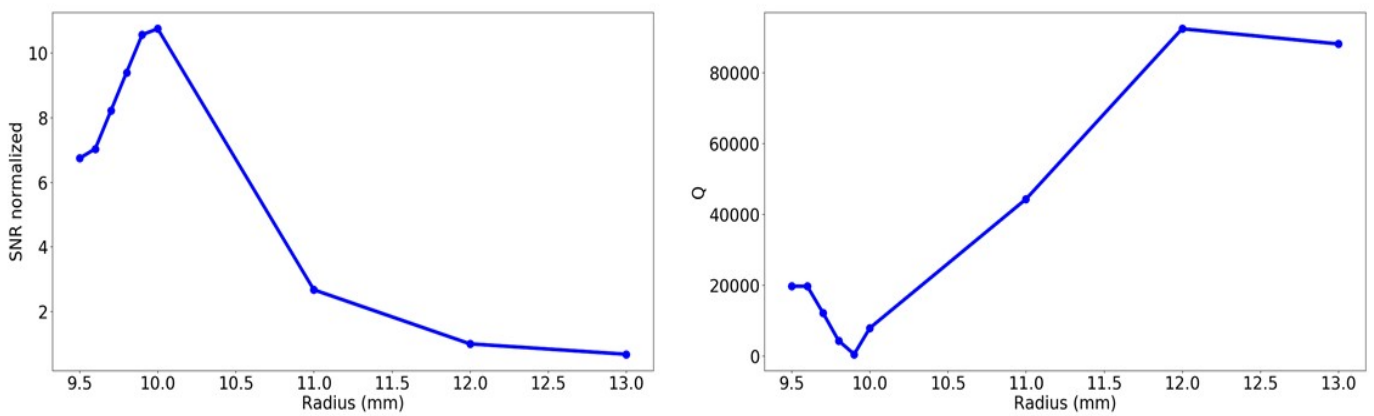

Fig. 2. FEM simulations of the influence of the resonator's radius on the system's signal to noise ratio and quality factor. The SNR is normalized with the SNR obtained at the best quality factor (radius equal to $12 \mathrm{~mm}$ ).

Finally, we designed a radial acoustical resonator that should lead to an overall QEPAS sensitivity equivalent to the state of the art of QEPAS with longitudinal resonators. The use of such a resonator should facilitate industrialization through an optical ease of alignment and ease of mechanical positioning.
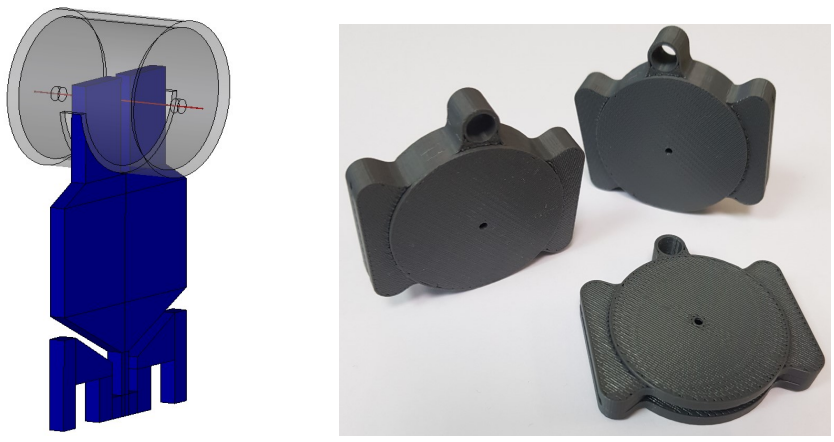

Fig. 3. Sketch of our new radial acoustical resonator (left) and first 3D-printed realizations (right).

Such radial resonators have been 3D printed (Figure 3) to confirm the predictions of FEM simulations by measurements, especially regarding the optimal radius.

\section{References}

[1] C. F. Dewey, R. D. Kamm, and C. E. Hackett, "Acoustic amplifier for detection of atmospheric pollutants," Appl. Phys. Lett., vol. 23, no. 11 , pp. $633-635,1973$.

[2] B. Kost, B. Baumann, M. Germer, M. Wolff, and M. Rosenkranz, "Numerical shape optimization of photoacoustic resonators," Appl. Phys. B Lasers Opt., vol. 102, no. 1, pp. 87-93, 2011.

[3] A. Miklós, P. Hess, and Z. Bozóki, "Application of acoustic resonators in photoacoustic trace gas analysis and metrology," Rev. Sci. Instrum., vol. 72, no. 4, pp. 1937-1955, 2001.

[4] A. A. Kosterev, Y. A. Bakhirkin, R. F. Curl, and F. K. Tittel, “Quartz-enhanced photoacoustic spectroscopy,” Opt. Lett., vol. 27, no. 21, p. $1902,2002$.

[5] V. Spagnolo, P. Patimisco, A. Sampaolo, and M. Giglio, "Recent advances in quartz enhanced photoacoustic sensing," vol. 2017, no. January, pp. 7-8, 2017.

[6] H. Yi, W. Chen, S. Sun, K. Liu, and T. Tan, "T-shape microresonator-based high sensitivity quartz-enhanced photoacoustic spectroscopy sensor," vol. 20, no. 8, pp. 9187-9196, 2012.

[7] G. Aoust, R. Levy, B. Verlhac, and O. Le Traon, "Optimal quality factor for tuning forks in a fluid medium," Sensors Actuators, $A$ Phys., vol. 243, pp. 134-138, 2016.

[8] V. Zeninari, R. Vallon, C. Risser, and B. Parvitte, "Photoacoustic Detection of Methane in Large Concentrations with a Helmholtz Sensor: Simulation and Experimentation," Int. J. Thermophys., vol. 37, no. 1, pp. 1-11, 2016.

[9] M. Duquesnoy, G. Aoust, J. M. Melkonian, R. Lévy, M. Raybaut, and A. Godard, "Quartz enhanced photoacoustic spectroscopy based on a custom quartz tuning fork," Sensors (Switzerland), vol. 19, no. 6, pp. 1-10, 2019. 